\title{
Strategies to Circumvent Testosterone Surge and Disease Flare in Advanced Prostate Cancer: Emerging Treatment Paradigms
}

\author{
Venkata K. Pokuri, MD; Houman Nourkeyhani, MD; Bodie Betsy, MSN, RN, NP; Laurie Herbst, PA; \\ Marcus Sikorski, MSN, RN, NP; Edward Spangenthal, MD; Andrew Fabiano, MD; and Saby George, MD
}

\begin{abstract}
The testosterone surge and disease flare is a feared complication from initiation of gonadotropin-releasing hormone (GnRH) agonist treatment in advanced prostate adenocarcinoma. It is a common practice to start an average 7-day pretreatment regimen with an antiandrogen agent before initiating GnRH agonist therapy, to circumvent disease flare from testosterone surge. However, this might not be the best strategy and can be harmful, especially in patients at high risk of imminent organ damage from minimal testosterone surge. Surgical castration is a simple and cost-effective method that should be considered in these scenarios. But most patients refuse this procedure because of the permanent and psychologic impact of surgery. Novel GnRH antagonists, such as degarelix, and cytochrome P450 17 (CYP17) enzyme inhibitors, such as ketoconazole, achieve castrate-equivalent serum testosterone levels much faster than traditional GnRH agonists without the need for coadministration of antiandrogens. This article reports on 3 cases of impending oncologic emergencies in advanced prostate adenocarcinoma treated promptly with degarelix and ketoconazole without any disease flare related to testosterone surge. In the setting of symptomatic hormone-naïve metastatic prostate cancer, the authors suggest clinical trials using abiraterone, orteronel, and other newer agents that target the CYP17 axis (eg, ketoconazole) for fine-tuning the emergent medical castration methods and avoiding the dangers from the flare phenomenon. (J Natl Compr Canc Netw 2015; 13:e49-e55)
\end{abstract}

Despite improvements in therapy for prostate cancer, oncologic emergencies, usually encountered in patients with advanced disease, continue to present challenges to the practicing oncologist. Common presentations include spinal cord compression from bone metastasis and bladder outlet obstruction from an enlarged prostate. Pericardial tamponade from pericardial metastasis is rare and has been reported in advanced prostate cancer. ${ }^{1}$ Although the availability of better therapies has made these difficult problems increasingly manageable, prompt recognition and the institution of adequate therapy are essential. Androgen deprivation therapy (ADT) with gonadotropin-releasing hormone (GnRH) agonists has remained the mainstay of prostate cancer treatment for years. However, GnRH agonists, when used alone in these oncologic emergencies, can cause initial testosterone surge and worsen the symptoms caused by disease flare. The risk of flare is greatly affected by the volume of disease present at the initiation of GnRH therapy and by the site of metastasis. ${ }^{2}$ This article presents 3 cases of impending nerve root compression, spinal cord compression, and cardiac tamponade, demonstrating effective therapeutic approaches to avoid testosterone-related disease flare.

\section{Case Presentations}

\section{Case 1}

From Roswell Park Cancer Institute, Buffalo, New York.

Submitted June 6, 2014; accepted for publication

December 3, 2014.

Dr. George has disclosed that he has received research funding from GlaxoSmithKline, and is a consultant and advisory board member for AVEO Pharmaceuticals, Inc., sanofi-aventis US LLC, Bayer HealthCare/Algeta, and Novartis. The remaining authors have disclosed that they have no financial interests, arrangements, affiliations, or commercial interests with the manufacturers of any products discussed in this article or their competitors.

Correspondence: Saby George, MD, Genitourinary Program, Roswell Park Cancer Institute, Elm \& Carlton Streets, Buffalo, NY 14263. E-mail: Saby.George@RoswellPark.org
A 45-year-old Caucasian man presented with fatigue, weight loss (30 pounds in 2 months), and back pain, which he had experienced for a few months. The back pain was located between his shoulder blades, radiating to his right arm, and associated with some numbness. MRI of his spine revealed indeterminate lesions in cervical and thoracic vertebrae. He also had extensive cervical and soft tissue signal abnormalities in the inter- 
spinous region, without cord compression, suggestive of metastatic disease (Figure 1). The lower right cervical nerve roots (C5-7) appeared to be compromised by enhancing foraminal, paraspinal soft tissue. Subsequent CT-guided biopsy of the thoracic vertebral lesion revealed metastatic adenocarcinoma of prostatic origin and his prostate-specific antigen (PSA) level was elevated at $149 \mathrm{ng} / \mathrm{mL}$. His local oncologist started him on bicalutamide (antiandrogen) for 2 weeks followed by a leuprolide ( $\mathrm{GnRH}$ agonist) injection. A week after initiation of the GnRH agonist, the patient noticed worsening weakness in his right upper extremity associated with increasing numbness. He presented to Roswell Park Cancer Institute for a second opinion, and reported that he was unable to move the ring and little fingers on his right hand. He was immediately started on high-dose ketoconazole (400 mg orally 3 times daily) along with hydrocortisone. One week later, his symptoms were stable. However, weakness persisted in the right upper extremity. A 240-mg loading dose of degarelix (a GnRH antagonist) was then administered subcutaneously. Ketoconazole was discontinued and hydrocortisone was tapered. He received another dose of degarelix ( $80 \mathrm{mg}$ subcutaneously) 4 weeks later. His motor strength improved in approximately 2 months, at which point he was transitioned to a GnRH agonist.

\section{Case 2}

A 75-year-old African American man was initially diagnosed with localized, Gleason grade $7(3+4)$ prostatic adenocarcinoma. He underwent radical

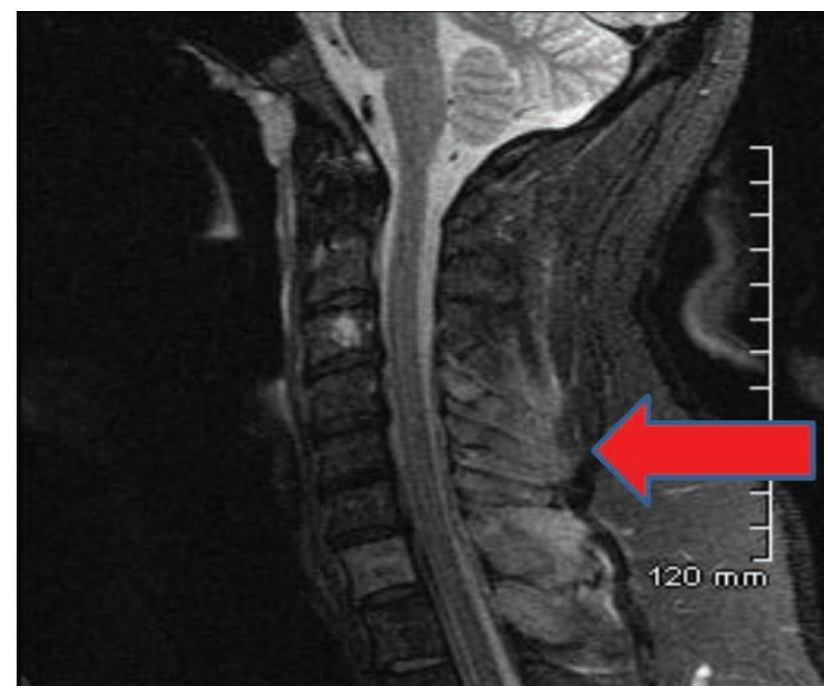

Figure 1 Extensive soft tissue abnormality (arrow) in lower cervical interspinous area, without spinal cord compression, suggestive of metastasis. prostatectomy, which revealed $\mathrm{pT} 2 \mathrm{cNO}$ disease. $\mathrm{He}$ had no extra prostatic extension or seminal vesicle involvement and margins were negative. His PSA level was $10 \mathrm{ng} / \mathrm{mL}$ before surgery and decreased to less than $0.01 \mathrm{ng} / \mathrm{mL}$ after prostatectomy. After surgery he was lost to follow-up. Seven years later, he presented with 3 weeks of gait unsteadiness associated with back pain and a 15-pound unintentional weight loss. The pain he was experiencing was intermittent in the lower back radiating down to the back of his legs and to the front of his abdomen in a band-like fashion. He denied any bowel or bladder incontinence. On examination, he experienced moderate tenderness to palpation in the lower thoracic spine and his gait was guarded, especially when attempting to walk in a straight line. Otherwise, no gross focal neurologic deficits were noticed. Blood work was unremarkable, except for elevated alkaline phosphatase and PSA levels $(9.8 \mathrm{ng} / \mathrm{mL})$. CT scan of the abdomen showed a blastic lesion at the T9 vertebral level with extension to the pedicles and spinous process. Central canal impingement was seen on MRI (Figure 2). Additionally, extension of the tumor into the adjacent left posterior 9 th rib at the costovertebral junction was seen. Bone scan (Figure 2) revealed widespread bony metastases, most prominent in the thoracic spine. In view of the impending cord compression and his prior noncompliance, the patient was offered emergent bilateral orchiectomy to attain rapid decline in testosterone levels. However, he refused to undergo surgery. He was given the GnRH antagonist degarelix (240 mg subcutane-

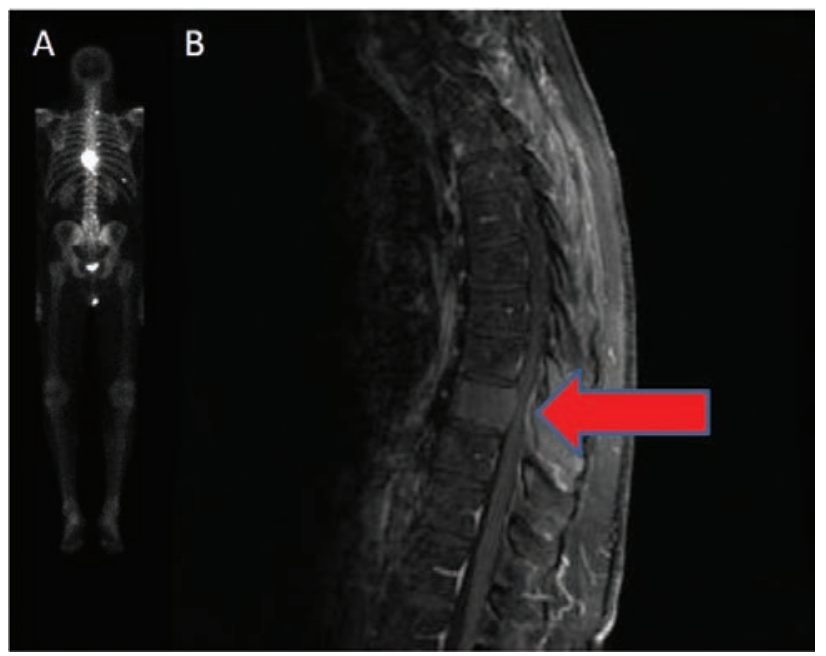

Figure 2 (A) Bone scan showing increased activity at the lower (T8T10) thoracic region. (B) T1-weighted sagittal MRI demonstrating a T9centered metastatic focus causing some spinal cord compression (arrow). 
ously). Subsequently, his pain resolved and his gait improved in 2 days. His PSA level decreased to 0.7 $\mathrm{ng} / \mathrm{mL}$ and he underwent thoracic laminectomy at the levels of T8, T9, and T10, with removal of an epidural-based tumor in 2 weeks. Pathology revealed adenocarcinoma of prostatic origin, with immunohistochemical staining positive for PSA and PSAP (prostate-specific acid phosphatase). After surgery, he was much more comfortable and able to ambulate and perform activities of daily living independently.

\section{Case 3}

A 41-year-old Caucasian male presented with worsening generalized bone pain, fatigue, and weight loss experienced over a few months. CT scan showed diffuse bony metastatic lesions (involving spine, ribs, and appendicular skeleton), lymphadenopathy (mediastinal and retroperitoneal), and moderate pericardial effusion (Figure 3). Workup also revealed an elevated PSA level $(9295 \mathrm{ng} / \mathrm{mL})$ and elevated alkaline phosphatase. Subsequent prostate biopsy revealed high-risk adenocarcinoma with a Gleason grade $9(4+5)$. He was admitted to the hospital 3 days later, after presenting with worsening bone pain and dyspnea on exertion. He denied bowel or bladder incontinence. On examination, muscle strength was slightly decreased in lower extremities, but no obvious neurologic deficits were noted. He was found to have distended neck veins with prominent jugular venous pulsations. MRI of the spine did not reveal signs of cord compression. However, transthoracic echocardiogram revealed moderate pericardial effusion with hemodynamic signs of early tamponade,

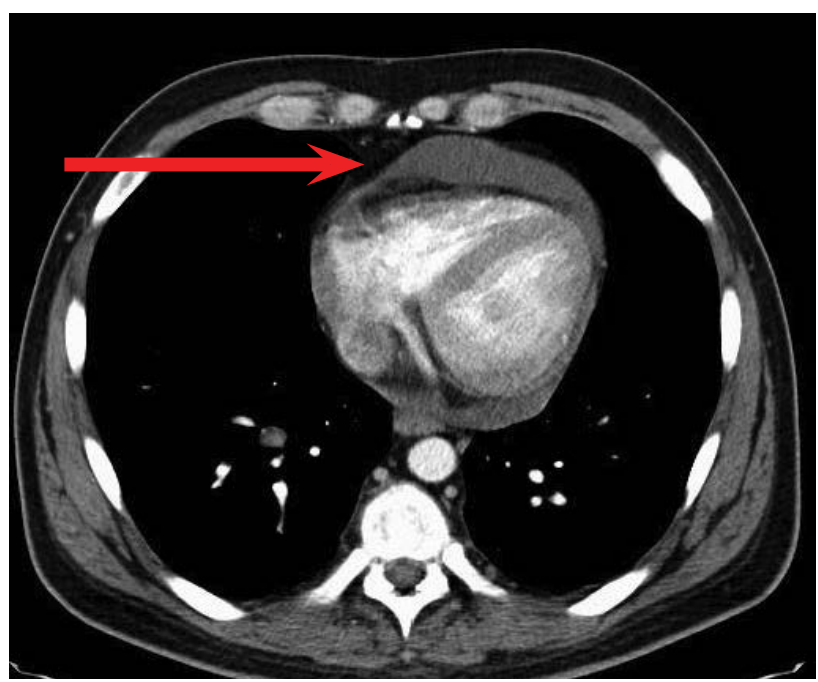

Figure 3 CT scan revealing moderate pericardial effusion (arrow). along with echogenic densities within pericardium, consistent with metastatic disease (Figure 4).

In view of early tamponade, he was given ketoconazole (400 mg 3 times daily) along with hydrocortisone. The next day, he was taken to the operating room to evaluate the possibility of pericardial drain placement, and bedside cardiac ultrasound revealed very minimal pericardial fluid, precluding the need for drainage. Additionally, within 24 hours of ketoconazole initiation, his testosterone levels dropped to $60 \mathrm{ng} / \mathrm{dL}$ and PSA level decreased by approximately 50\% (5304 ng/mL). Subsequently, his pain and breathing improved and he was discharged uneventfully. He was later transitioned to GnRH agonist without any disease flare. Currently, he is alive 3 years after the initial presentation and has evolved through different treatments.

\section{Discussion}

The principle of ADT as a treatment for advanced prostatic adenocarcinoma was initially described in $1941 .^{3}$ Authors demonstrated that lowering testosterone through surgical castration or injection of estrogens induces a major regression of prostate adenocarcinoma and rapid relief of symptoms from metastatic disease. A few years later, the discovery of the GnRH receptor and synthesis of the first GnRH analog established ADT as the key systemic treatment against prostate adenocarcinoma. ${ }^{4}$ In the modern era, clinicians are still relying on this principle in the first-line treatment of metastatic hormonesensitive prostate cancer.

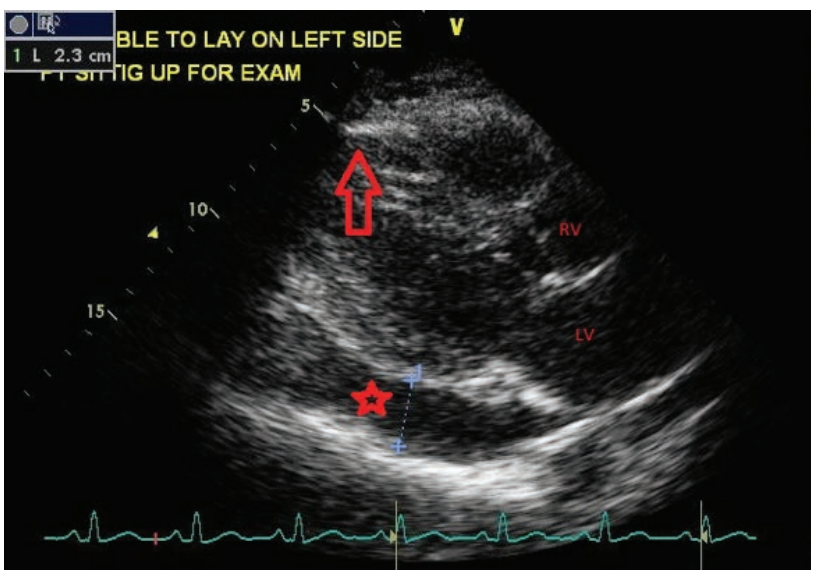

Figure 4 Transthoracic echocardiogram showing circumferential pericardial effusion (star) and echogenic density attached to visceral pericardium (arrow), consistent with metastatic disease. 
ADT can be accomplished either surgically (bilateral orchiectomy) or medically (using $\mathrm{GnRH}$ agonist or antagonist; Table 1). Both methods are equally effective and achieve a reduction in PSA levels, overall tumor burden, and complications, such as pathologic fractures, obstructive uropathy, or spinal cord compression. ${ }^{5}$ Although surgical orchiectomy has fallen out of favor in Europe and North America, mostly because of concerns about the permanent and psychologic impact of surgery, ${ }^{6}$ it remains the initial preferred treatment in many parts of the world and is also viewed as a treatment option should an immediate decrease in testosterone be warranted. Castrate levels of testosterone are normally achieved in 3 to 12 hours (mean, 8.6 hours) with bilateral orchiectomy. ${ }^{3,7}$ It is a simple procedure that could be performed under local anaesthesia. It is very cost-effective and patients do not need to be on lifelong GnRH agonist therapy afterwards. Surgical castration may actually be more appealing in patients whose adherence to medical treatment is poor (such as in Case 2).

Medical castration relies on the modulation of the hypothalamic-pituitary axis through the use of GnRH agonists. Synthetic GnRH analogs are preferred over natural-occurring molecules for their increased affinity for the GnRH receptor. GnRH release from the hypothalamus stimulates the pituitary gland to secrete luteinizing hormone (LH) and follicle-stimulating hormone (FSH). LH then stimulates Leydig cells in the testes to secrete testosterone. Release of LH is in turn controlled by $\mathrm{GnRH}$ secretion from the hypothalamus, which normally occurs in a regular, pulsatile manner. GnRH agonists override this pulsatile control of the pituitary by providing continuous stimulation, which eventually leads to downregulation of pituitary GnRH receptors, a reduction in $\mathrm{LH}$ production, and therefore a sup-

\begin{tabular}{|lll|}
\hline $\begin{array}{l}\text { Table } 1 \\
\text { Modalities } \\
\text { Mormacodynamics of Various ADT }\end{array}$ \\
$\begin{array}{lll}\text { Time to Attain } \\
\text { Castrate Level } \\
\text { ADT Strategy }\end{array}$ & $\begin{array}{l}\text { Risk of Initial } \\
\text { Testosterone }\end{array}$ & Testosterone Surge \\
\hline GnRH analogs & $2-4 \mathrm{wk}^{10,16}$ & Yes \\
Degarelix & $3 \mathrm{~d}^{16}$ & No \\
Ketoconazole & $24 \mathrm{~h}^{25,26}$ & No \\
Surgical castration & $3-12 \mathrm{~h}^{3,7}$ & No \\
\hline
\end{tabular}

Abbreviations: ADT, androgen deprivation therapy; GnRH, gonadotropin-releasing hormone. pression of testosterone levels. However, the use of a GnRH receptor agonist initially causes stimulation of the entire axis, resulting in a surge of testosterone.

The initial transitory testosterone surge is the cause of what has been termed the flare phenomenon. This surge begins approximately 2 or 3 days after the initial injection of GnRH agonist and lasts through approximately the first week of therapy. ${ }^{2}$ Median serum testosterone levels can increase 2 -fold or more above baseline during this period. ${ }^{8}$ Rapid increase in tumor burden can occur with the testosterone surge and is dangerous in scenarios of impending spinal cord compression from bone metastasis and bladder outlet obstruction from an enlarged prostate. Sudden deaths from disease flare also have been reported in the literature. ${ }^{9}$

Wide variation exists in the reported frequency of flare responses $(4 \%-63 \%)$ from luteinizing hormone-releasing hormone (LHRH) analogs during the initial treatment of patients with severe metastatic disease, ${ }^{2}$ mainly because of the confusion about biochemical versus clinical flare responses. However, Kuhn et al, ${ }^{10}$ who clearly distinguished biochemical from clinical flare reactions, reported a high frequency of $63 \%$. Furthermore, patients at risk for clinical flare are mostly those with widespread symptomatic metastatic disease, rather than those with minimal metastasis or PSA progression only.

It is a common practice to give antiandrogens like bicalutamide, either concurrently or preceding $\mathrm{GnRH}$ agonists, and be continued in combination for at least 7 days to prevent a clinically significant flare from testosterone surge. ${ }^{10-12}$ However, this strategy is not always effective. Oh et $\mathrm{al}^{13}$ analyzed 1566 patients from Veterans Affairs hospitals with metastatic prostate cancer and showed that antiandrogen therapy given before $\mathrm{GnRH}$ agonists was not associated with differences in fractures, spinal cord compression, or bladder outlet obstruction. Antiandrogens might decrease the incidence of clinical flares but do not totally eliminate the occurrence of these flares in high-risk patients (and they do not result in lower testosterone levels). ${ }^{2}$ These agents might not be able to block all of the androgen receptors effectively, especially in cases with bulky metastatic disease associated with a high risk of imminent organ damage from minimal testosterone surge.

This is clearly illustrated in Case 1 , in which the patient presented with upper extremity neurologic deficits, secondary to metastatic disease involving 
the brachial plexus. His symptoms worsened with the antiandrogen/GnRH agonist strategy. He was then treated with the alternative strategy (ketoconazole/degarelix) to achieve rapid castration. However, it took almost 2 to 3 months for him to recover. Although his motor strength improved, it never returned to baseline, suggesting some amount of irreversible damage to the nerve plexus occurred, with the initiation of $\mathrm{GnRH}$ analog causing testosterone surge and subsequent disease flare.

Unlike GnRH agonists that initially stimulate $\mathrm{GnRH}$ receptors before leading to hypogonadism, $\mathrm{GnRH}$ antagonists rapidly and directly inhibit the release of androgens. Therefore, no flare is associated with these agents and no coadministration of antiandrogen is necessary. Abarelix was the first GnRH antagonist available clinically for prostate cancer treatment. However, it was withdrawn from the US market because of concerns about immediate systemic allergic reactions from histamine release. ${ }^{14}$ Degarelix was a third-generation $\mathrm{GnRH}$ antagonist that was synthetically modified with a view toward reducing this histamine-releasing activity. It was the first GnRH antagonist approved by the FDA in 2008. ${ }^{14,15}$ The pivotal trial (CS21 trial) that led to its approval was an open-label study that randomized 610 patients ${ }^{16}$ to 1 of the 3 regimens: monthly $7.5-\mathrm{mg}$ leuprolide or to 1 of 2 degarelix regimens (a starting loading dose of $240 \mathrm{mg}$ degarelix followed by monthly maintenance doses of 80 or $160 \mathrm{mg}$ ). Patients in the leuprolide group could receive bicalutamide flare protection at the discretion of the investigator. In this trial, 144 (81\%) patients in the leuprolide group experienced a testosterone surge, including 17 of the 23 patients (74\%) who received concomitant bicalutamide. None of the patients in the degarelix arms (240/80-mg and 240/160-mg arms) experienced a testosterone surge. Both degarelix and leuprolide achieved the same level of testosterone suppression; $96 \%$ of patients receiving degarelix had a testosterone level of $50 \mathrm{ng} / \mathrm{dL}$ or lower within 3 days. This novel GnRH antagonist can achieve castrate-equivalent serum testosterone levels (defined as a serum testosterone level $\leq 50 \mathrm{ng} / \mathrm{dL}$ ) in 3 days versus 2 to 4 weeks with $\mathrm{GnRH}$ agonists. ${ }^{10,16}$ In line with the testosterone results, PSA levels also declined more rapidly in the degarelix arms at days 14 and 28. ${ }^{16}$

The rapid reductions in testosterone and PSA levels with degarelix suggest that it might be particu- larly appropriate for patients requiring fast symptom relief (either from primary disease or metastasis) or volume reduction (similar to described cases). Anderson et $\mathrm{al}^{17}$ from Europe initiated a 12-week phase IIIb study in men with predominantly locally advanced prostate cancer and highly symptomatic (lower urinary tract symptoms [LUTS]) disease, comparing degarelix (240/80) with goserelin plus bicalutamide (G/B). The primary end point was change in International Prostate Symptom Score (IPSS) at week 12 compared with baseline. This study was stopped early because of recruitment difficulties, and only $40 \mathrm{pa}$ tients were treated. Despite this, degarelix was noninferior to G/B for the IPSS at week 12 and superior in the per-protocol analysis $(P=.04)$. Furthermore, degarelix was associated with better improvements in prostate volume and quality of life. Axcrona et $\mathrm{al}^{18}$ from Norway conducted a similar study in 182 patients with LUTS from locally advanced prostate cancer. Patients were randomized to monthly degarelix (240/80 mg) or G/B for 12 weeks. Reduction of total prostate volume (TPV; measured monthly via transrectal ultrasonogram) was the primary end point and was not different between both arms at week 12 , meeting the predefined noninferiority criterion. However, degarelix showed superiority in LUTS relief in symptomatic patients, highlighting its action on extrapituitary $\mathrm{GnRH}$ receptors in the bladder and/or prostate. Mason et $\mathrm{al}^{19}$ showed similar noninferiority of degarelix (compared with $G / B$ ) in prostate shrinkage but more pronounced LUTS relief in the neoadjuvant setting before local radiation. These studies underscore the superiority of degarelix in controlling symptoms from locally advanced prostate cancer.

CYP17 inhibitors such as ketoconazole (antifungal agent) block the synthesis of androgens by both gonads and adrenal gland through inhibition of the enzyme 17, 20-lyase. ${ }^{20-22}$ It also has a direct cytotoxic effect on prostatic cancer cells in vitro. ${ }^{23}$ High-dose ketoconazole (400 mg administered orally every 8 hours) is studied in prostate cancer and has been shown to reduce bone pain, need for analgesic use, and serum prostatic acid phosphatase levels. ${ }^{24}$ It has also been shown to cause a decline in serum testosterone level within 30 minutes, produce castrate-equivalent serum testosterone levels within 24 hours of initiation, and a $90 \%$ reduction in testosterone level by 48 hours. ${ }^{25-27}$ It is often given in 
combination with oral hydrocortisone to prevent addisonian crisis. However, it is not FDA approved for prostate cancer, because safety and effectiveness have not been established. The most common side effects with prolonged use of high-dose ketoconazole include nausea, vomiting, liver toxicity, skin reactions, and a potential risk of adrenal suppression. ${ }^{28-30}$

Given the prompt and efficient antiandrogenic properties of ketoconazole, it has been used successfully in cases of metastatic prostatic carcinoma with impending neurologic injury and in patients with acute renal failure caused by bilateral ureteral obstruction from advanced prostatic carcinoma. ${ }^{31,32}$ The terminal half-life elimination for ketoconazole is 8 hours and its inhibitory effect is completely reversible. Testosterone levels and adrenal hormone levels return to normal within 24 hours after cessation of this drug. ${ }^{33,34}$ Therefore, if loading degarelix (240 mg) is also given with ketoconazole in these clinical situations, ketoconazole can be stopped in 3 to 4 days, because degarelix will maintain the castrate-level testosterone by 3 days (of note, degarelix half-life with loading dose is approximately 53 days). The current model that the authors use for emergent medical castration is a loading dose of $240 \mathrm{mg} \mathrm{sub}$ cutaneous degarelix along with oral ketoconazole at $400 \mathrm{mg} 3$ times a day (hydrocortisone at $20 \mathrm{mg}$ in the morning and $10 \mathrm{mg}$ in the evening is given to prevent adrenal insufficiency). Ketoconazole can be discontinued within 3 to 4 days (and long-term side effects can be avoided) if concurrent loading degarelix is injected on day 1 . The hydrocortisone can usually be discontinued without any adrenal complications. If there are signs of adrenal insufficiency, hydrocortisone taper can be considered.

Oral steroidal progestogens with additional antiandrogenic and antigonadotropic properties such as cyproterone acetate (CPA $)^{35,36}$ and chlormadinone acetate (CMA) $)^{35,37}$ were also studied to evaluate their effect on testosterone suppression. Appu et $\mathrm{al}^{38}$ showed that CPA administration before $\mathrm{GnRH}$ agonist injection achieved a castrate level of testosterone in 1 week; however, it did not eliminate the surge in serum testosterone when a GnRH agonist was administered..$^{38}$ A Japanese study showed that CMA was capable of preventing testosterone flare in advanced prostate cancer. ${ }^{39}$

\section{Conclusions}

Testosterone surge and disease flare occur at varied frequency when LHRH agonists are used for the initial treatment of symptomatic patients with widely metastatic prostate adenocarcinoma, depending on the volume of disease. These side effects are often dangerous, and sudden deaths have been reported from the clinical flare response. A crucial factor that determines irreversible damage from a potential flare phenomenon could be the metastatic sites of disease, including paraspinal cord lesions, pericardial metastasis with effusion, and the burden of disease. It is of utmost importance to recognize such impending oncologic emergencies and attain rapid medical castration through avoiding flare phenomenon. Surgical castration is a simple, cost-effective procedure that can achieve castrate levels of testosterone in a few hours, and may actually be more appropriate in patients whose adherence to medical treatment is poor. However, this procedure has fallen out of favor in the United States because of psychologic concerns with bilateral orchiectomy. The common paradigm of $\mathrm{GnRH}$ agonist treatment preceded by 1 to 2 weeks of antiandrogen should not be encouraged in these situations. Antiandrogens might decrease the incidence of clinical flares but do not totally eliminate occurrence in high-risk patients. The authors currently use high doses of oral ketoconazole for emergent castration and often administer $\mathrm{GnRH}$ antagonist (degarelix) loading dose on the first day, to allow for the quick discontinuation of ketoconazole.

The described cases underscore the ease of administering degarelix and ketoconazole and the rapidity of lowering androgen levels without causing testosterone surge, compared with the GnRH agonist/antiandrogen strategy. In the setting of symptomatic hormone-naive metastatic prostate cancer, the authors suggest clinical trials using abiraterone, orteronel (TAK-700), and other newer agents that target the CYP17 axis (eg, ketoconazole) for finetuning the emergent medical castration methods and avoiding the dangers from the flare phenomenon.

\section{References}

1. Minagawa T, Murata $Y$, Uchikawa S, Uehara T. Malignant pericardial tamponade in a patient with hormone-refractory prostate cancer. Int J Clin Oncol 2010;15:101-103.

2. Bubley GJ. Is the flare phenomenon clinically significant? Urology 2001;58(2 Suppl 1):5-9. 
3. Huggins C, Stevens RE Jr, Hodges CV. Studies on prostate cancer. II. The effect of castration on advanced carcinoma of the prostate gland. Arch Surg 1941;43:209-223.

4. Tolis G, Ackman D, Stellos A, et al. Proc Natl Acad Sci U S A 1982;79:1658-1662.

5. Loblaw DA, Mendelson DS, Talcott JA, et al. American Society of Clinical Oncology recommendations for the initial hormonal management of androgen-sensitive metastatic, recurrent, or progressive prostate cancer. J Clin Oncol 2004;22:2927-2941.

6. Cassileth BR, Soloway MS, Vogelzang NJ, et al. Patients' choice of treatment in stage D prostate cancer. Urology 1989;33(5 Suppl):57-62.

7. Lin BJ, Chen KK, Chen MT, Chang LS. The time for serum testosterone to reach castrate level after bilateral orchiectomy or oral estrogen in the management of metastatic prostatic cancer. Urology 1994;43:834-837.

8. Agarwal DK1, Costello AJ, Peters J, et al. Differential response of prostate specific antigen to testosterone surge after luteinizing hormone-releasing hormone analogue in prostate cancer and benign prostatic hyperplasia. BJU Int 2000;85:690-695.

9. Thompson IM, Zeidman EJ, Rodriguez FR. Sudden death due to disease flare with luteinizing hormone-releasing hormone agonist therapy for carcinoma of the prostate. J Urol 1990;144:1479-1480.

10. Kuhn JM, Billebaud T, Navratil H, et al. Prevention of the transient adverse effects of a gonadotropin-releasing hormone analogue (buserelin) in metastatic prostatic carcinoma by administration of an antiandrogen (nilutamide). N Engl J Med 1989;321:413-418.

11. Labrie F, Dupont A, Belanger A, Lachance R. Flutamide eliminates the risk of disease flare in prostatic cancer patients treated with a luteinizing hormone-releasing hormone agonist. J Urol 1987;138:804-806.

12. Schulze H, Senge T. Influence of different types of antiandrogens on luteinizing hormone-releasing hormone analogue-induced testosterone surge in patients with metastatic carcinoma of the prostate. J Urol 1990;144:934-941.

13. Oh WK, Landrum MB, Lamont EB, et al. Does oral antiandrogen use before leuteinizing hormone-releasing hormone therapy in patients with metastatic prostate cancer prevent clinical consequences of a testosterone flare? Urology 2010;75:642-647.

14. Rick FG, Block NL, Schally AV. An update on the use of degarelix in the treatment of advanced hormone-dependent prostate cancer. Onco Targets Ther 2013;6:391-402.

15. Broqua P, Riviere PJ, Conn PM, et al. Pharmacological profile of a new, potent, and long-acting gonadotropin-releasing hormone antagonist: degarelix. J Pharmacol Exp Ther 2002;301:95-102.

16. Klotz L, Boccon-Gibod L, Shore ND, et al. The efficacy and safety of degarelix: a 12-month, comparative, randomized, open-label, parallel-group phase III study in patients with prostate cancer. BJU Int 2008;102:1531-1538.

17. Anderson J, Al-Ali G, Wirth $\mathrm{M}$, et al. Degarelix versus goserelin (+ antiandrogen flare protection) in the relief of lower urinary tract symptoms secondary to prostate cancer: results from a phase IIIb study (NCT00831233). Urol Int 2013;90:321-328.

18. Axcrona K, Aaltomaa S, da Silva CM, et al. Androgen deprivation therapy for volume reduction, lower urinary tract symptom relief and quality of life improvement in patients with prostate cancer: degarelix vs goserelin plus bicalutamide. BJU Int 2012;110:1721-1728.

19. Mason M, Maldonado Pijoan X, Steidle C, et al. Neoadjuvant androgen deprivation therapy for prostate volume reduction, lower urinary tract symptom relief and quality of life improvement in men with intermediateto high-risk prostate cancer: a randomised non-inferiority trial of degarelix versus goserelin plus bicalutamide. Clin Oncol (R Coll Radiol) 2013;25:190-196.

20. De Coster R, Caers I, Coene MC, et al. Effects of high dose ketoconazole therapy on the main plasma testicular and adrenal steroids in previously untreated prostatic cancer patients. Clin Endocrinol (Oxf) 1986;24:657664.

21. Santen RJ, Van den Bossche H, Symoens J, et al. Site of action of low dose ketoconazole on androgen biosynthesis in men. J Clin Endocrinol Metab 1983;57:732-736.

22. Trachtenberg J, Zadra J. Steroid synthesis inhibition by ketoconazole: sites of action. Clin Invest Med 1988;11:1-5.

23. Eichenberger T, Trachtenberg J, Toor P, Keating A. Ketoconazole: a possible direct cytotoxic effect on prostate carcinoma cells. J Urol 1989;141:190-191

24. Trachtenberg J, Pont A. Ketoconazole therapy for advanced prostate cancer. Lancet 1984;2:433-435.

25. Trachtenberg J. Ketoconazole therapy in advanced prostatic cancer. J Urol 1984;132:61-63

26. Heyns W, Drochmans A, van der Schueren E, Verhoeven G. Endocrine effects of high-dose ketoconazole therapy in advanced prostatic cancer. Acta Endocrinol (Copenh) 1985;110:276-283.

27. Trachtenberg J, Halpern N, Pont A. Ketoconazole: a novel and rapid treatment for advanced prostatic cancer. J Urol 1983;130:152-153.

28. Polsen JA, Cohen PR, Sella A. Acquired cutaneous adherence in patients with androgen-independent prostate cancer receiving ketoconazole and doxorubicin: medication-induced sticky skin. J Am Acad Dermatol 1995;32:571-575.

29. Small EJ, Baron AD, Fippin L, Apodaca D. Ketoconazole retains activity in advanced prostate cancer patients with progression despite flutamide withdrawal. J Urol 1997;157:1204-1207.

30. Small EJ, Baron A, Bok R. Simultaneous antiandrogen withdrawal and treatment with ketoconazole and hydrocortisone in patients with advanced prostate carcinoma. Cancer 1997;80:1755-1759.

31. Bamberger $\mathrm{MH}$, Lowe FC. Ketoconazole in initial management and treatment of metastatic prostate cancer to spine. Urology 1988;32:301303.

32. Agarwal MM, Singh SK, Acharya NC, et al. Non-interventional management of obstructive acute renal-failure in hormone-naive prostate cancer. Can J Urol 2007;14:3580-3582.

33. Pont A, Williams PL, Azhar S, et al. Ketoconazole blocks testosterone synthesis. Arch Intern Med 1982;142:2137-2140.

34. Sonino N. The use of ketoconazole as an inhibitor of steroid production. N Engl J Med 1987;317:812-818.

35. Raudrant D, Rabe T. Progestogens with antiandrogenic properties. Drugs 2003;63:463-492.

36. DonaldRA, EspinerEA, Cowles RJ, FazackerleyJE. The effect of cyproterone acetate on the plasma gonadotrophin response to gonadotrophin releasing hormone. Acta Endocrinol (Copenh) 1976;81:680-684

37. Chassard D, Schatz B. The antigonadrotropic activity of chlormadinone acetate in reproductive women [in French]. Gynecol Obstet Fertil 2005;33:29-34.

38. Appu S, Lawrentschuk N, Grills RJ, Neerhut G. Effectiveness of cyproterone acetate in achieving castration and preventing luteinizing hormone releasing hormone analogue induced testosterone surge in patients with prostate cancer. J Urol 2005;174:140-142.

39. Sato N, Kotake T, Masai M, Sakai S, Ito H. Preventive effect of chlormadinone acetate on flare-up phenomenon in advanced prostate cancer administered with a luteinizing hormone-releasing hormone analogue [in Japanese]. Hinyokika Kiyo 2000;46:1-7. 